\title{
1 Restriction enzyme selection dictates detection range sensitivity in chromatin \\ 2 conformation capture-based variant-to-gene mapping approaches
}

4 Chun Su${ }^{1}$, Matthew C. Pahl ${ }^{1}$, Struan F. A. Grant ${ }^{1,2,3,4 \star}$, and Andrew D. Wells ${ }^{5,6 *}$

$5 \quad{ }^{*}$ Co-leads

$6{ }^{1}$ Division of Human Genetics, The Children's Hospital of Philadelphia, 3615 Civic Center Boulevard,

7 Philadelphia, PA

$8{ }^{2}$ Department of Pediatrics, Perelman School of Medicine, University of Pennsylvania, 3615 Civic Center

9 Boulevard, Philadelphia, PA

$10{ }^{3}$ Division of Diabetes and Endocrinology, The Children's Hospital of Philadelphia, 3615 Civic Center

11 Boulevard, Philadelphia, PA

$12{ }^{4}$ Department of Genetics, Perelman School of Medicine, University of Pennsylvania, 3615 Civic Center

13 Boulevard, Philadelphia, PA

$14{ }^{5}$ Department of Pathology, The Children's Hospital of Philadelphia, 3615 Civic Center Boulevard,

15 Philadelphia, PA

$16{ }^{6}$ Department of Pathology and Laboratory Medicine, Perelman School of Medicine, University of

17 Pennsylvania, 3615 Civic Center Boulevard, Philadelphia, PA

18 


\section{Abstract}

21 Promoter-focused chromatin conformation techniques directly detect interactions between

22 gene promoters and distal genomic sequences, providing structural information relevant to gene

23 regulation without the excessive non-genic architectural data generated by full scale Hi-C. 3D

24 promoter 'interactome' maps are crucial for understanding how epigenomic features like histone

25 modifications and open chromatin, or genetic variants identified in genome wide association

26 studies (GWAS), contribute to biological function. However, variation in sensitivity between such

27 promoter-focused methods, principally due to restriction enzyme selection, has not been

28 systematically assessed. Here, we performed a head-to-head comparison between promoter

29 capture $\mathrm{C}(\mathrm{PCC})$ and promoter capture $\mathrm{Hi}-\mathrm{C}(\mathrm{PCHiC})$ with the respective 4 cutters Dpnll and

$30 \mathrm{Mbol}$ versus 6 cutter HindIII datasets from the same five cell types. While PCHiC generally

31 produces a higher signal to noise ratio for significant interactions in comparison to PCC, we

32 show that DpnII/Mbol detects more proximal interactions and shows little overlap with the HindIII

33 detection range. Promoter-interacting genomic regions mapped by 4-cutters are more enriched

34 for regulatory features and disease-associated genetic variation than 6-cutters maps,

35 suggesting that high-resolution maps better capture gene regulatory architectures than do lower

36 resolution approaches. 


\section{Main Text}

Chromatin conformation capture $(3 \mathrm{C})$ based methods combined with high-throughput deep sequencing are increasingly used to detect direct interactions between gene promoters and

41 distal genomic sequences. These techniques specifically enrich for promoter-containing DNA

42 fragments using complementary RNA oligomers, increasing the resolution and cost-efficiency of

43 detecting gene interactions compared to conventional $\mathrm{Hi}-\mathrm{C}$, which generates interaction data

44 across the whole, largely intergenic, genome. The resulting promoter contact maps are widely

45 used to assign distal regulatory elements to their target genes for a better understanding of

46 global regulation of gene expression ${ }^{1,2}$, and to connect human disease-associated variants to

47 their effector gene promoters following genome-wide association studies (GWAS) ${ }^{3-5}$.

Promoter capture C (PCC) is applied directly to $3 \mathrm{C}$ libraries, while promoter capture Hi-C (PCHiC) involves an additional streptavidin-biotin pulldown step before promoter capture

51 hybridization to enrich for genuine ligation products and increase the efficiency of sequencing

52 valid hybrid reads ${ }^{6}$. To compare the results of different promoter capture techniques, we 53 selected five distinct cell types with datasets available for both PCC and $\mathrm{PCHiC}^{1}$

54 (Supplemental Table 1). As previously estimated ${ }^{7}, \mathrm{PCHiC}$ read usage was 2 3 times more 55 efficient than PCC, as measured by the ratio of valid read pairs to mapped read pairs

56 (Supplemental Table 1). More dramatically, PCC data was enriched for valid read pairs that

57 mapped to a shorter range (Supplemental Figure 1 A), which may reflect the contribution of

58 undigested fragments instead of bona fide ligation products. The retention of partially digested

59 fragments in single-fragment analyses skews toward a higher percentage of short-range 60 significant interactions in PCC samples (Supplemental Figure 1 B), which may be artefactual.

61 To mitigate the influence of partial digestion in PCC, we concatenated neighboring fragments in 62 silico by binning valid reads from 4 fragments into one larger fragment, effectively filtering out 63 interactions potentially called due to partial digestion. This approach not only attenuated over- 
64 representation of short-range interactions in the PCC data (Supplemental Figure 1B), but also

65 increased the supporting read count per fragment to detect longer-distance interactions.

Another key variation in library preparation is the choice of restriction enzyme. The widely used restriction enzymes include HindIII with a 6 bp recognition site ${ }^{6,1,8,9}$ and Dpnll or Mbol with a 4 bp recognition site $4,10,3,11$. A computational Dpnll or Mbol restriction digest of the human genome predicts fragments 7 to 9 times smaller than a Hindll digest (Supplemental Figure 2 A), leading to the potential for higher-resolution interaction maps. In addition, the smaller

72 fragment size of Dpnll and Mbol leads to more precise annotation, pinpointing more than $75 \%$

73 of captured fragments (baits) to just one transcriptional start site (TSS), and resulting in a

74 median fragment size of $265 \mathrm{bp}$ in one fragment resolution (Supplemental Table 2). This

75 resolution equates to approximately two nucleosomes, or the size of a single chromatin

76 accessible region ${ }^{12}$, an important consideration if one wants to integrate with ATAC-seq or

77 ChIP-seq datasets. An example of this is shown at the GRAP2 and ENTHD1 genes in naïve

78 CD4 T cells, where DpnII PCC can resolve the interactomes of each promoter, but HindIII

79 PCHiC cannot (Supplemental Figure 2 B).

CHiCAGO is the most commonly used software for calling interactions for both PCC and

$82 \mathrm{PCHiC}^{13}$. With minor differences in parameter settings which take the fragment size difference

83 into account (see Methods), PCC and PCHiC, regardless of resolution, generate a comparable

84 number of total interactions, cis-trans interaction ratios, and bait-to-bait interaction ratios

85 (Supplemental Table 3). However, with the higher captured read-pair input, each significant 86 interaction is supported with a higher average number of reads in HindIII PCHiC (Supplemental 87 Figure 3), leading to a higher signal-to-noise ratio. 
One caveat in using CHiCAGO calls is that bait-to-bait interactions are not always called as "significant" at both baits, even though physically they must be bi-directional. This results from

91 CHiCAGO implementing bait-specific and counterpart-specific (other-end) factor scaling for

92 Brownian background estimation. The internal consistency between bait-to-bait interactions can

93 provide an intra-library metric for reproducibility. Compared to HindIII PCHiC, we observed a

94 significantly higher bi-directional (reproducible) bait-to-bait interaction ratio for Dpnll PCC

95 (Supplemental Figure 4 A, Supplemental Table 3). This likely results from less dispersed

96 bait-specific and other-end-specific scaling factors in the Dpnll PCC design (Supplemental

97 Figure 4 B), and suggests that Dpnll bait design results in more uniform hybridization across 98 baits.

While different capture techniques and restriction enzymes yield roughly comparable quality control metrics as outlined above, these approaches yield drastically different results with regard to the detection range of promoter interactions. The detection ranges of Mbol PCHiC, Dpnll PCC and HindIII PCHiC are all consistent with the size of mammalian topologically associating domains (TADs), which range from $100 \mathrm{~kb}$ to $5 \mathrm{Mb}$ with an average of $\sim 1 \mathrm{Mb}^{14}$. However, regardless of different capture techniques, while the HindllI detection range was between $150 \mathrm{~kb}$ and 2Mb, Dpnll-based and Mbol-based 4-cutter libraries detect many more proximal

107 interactions (Figure 1 A and B). Ninety percent of interactions detected by Dpnll PCC were 108 concentrated between $500 \mathrm{bp}$ and $500 \mathrm{~kb}$ (single fragment call: 500bp 300kb; 4 fragment call:

$1092 \mathrm{~kb} \sim 500 \mathrm{~kb}$ ), and the Mbol PCHiC detection distance was distributed from $6 \mathrm{kp}$ to $500 \mathrm{~kb}$

110 (Figure $1 \mathrm{~A}$ and $\mathbf{B}$ ). This difference in range resulted in only 10 to $40 \%$ of detected interactions

111 overlapping between HindIII and the 4-cutters, depending upon fragment resolution, library

112 quality and Chicago settings (Supplemental Table 4). We also compared the two methods at

113 the gene/promoter level, by comparing only baits harboring the same gene TSS to account for 114 differences in bait design and promoter annotation between the two approaches (Supplemental 
115 Table 5). Approximately $65 \%$ of genes were engaged in at least one significant interaction with

116 a distal region in both the DpnII PCC and HindllI PCHiC libraries, with twice as many DpnII-

117 specific interacting genes called compared to Hindlll (Figure $1 \mathbf{C}$, left panel). The set of

118 interactive genes detected by Mbol PCHiC vs. HindIII PCHiC is even less similar, with only $43 \%$

119 of genes having at least one significant distal interaction in both the Mbol PCHiC and HindIII

120 PCHiC CM libraries (Figure $1 \mathrm{C}$, right panel). To further explore the drivers behind the

121 resolution-specific genes, we compared the interaction distance for 4-cutter-specific interacting

122 genes to those common to both cutters, and found that 4-cutter-specific interacting genes

123 involved contacts at significantly shorter distances (Figure 1 D, two-tailed Wilcoxon sum rank

124 test). This suggests that the 4-cutter methods detect contacts that are too promoter-proximal to

125 be detected in HindIII PCHiC datasets. Indeed, we could find a myriad of sub-150kb promoter

126 contacts revealed by Dpnll-resolution PCC that were not detected by HindIII PCHiC. For

127 example, Dpnll PCC detected dozens of interactions within $150 \mathrm{~kb}$ of the baited SERBP1

128 promoter in naïve B cells, including open regions residing in introns of IL12RB2. However, those

129 interactions were not detected in HindIII PCHiC (Figure 1E). Conversely, DpnII PCC with 4-

130 fragment binning failed to detect very long-range interactions detected with HindIII (>500kbp).

131 However, by increasing fragment concatenation levels further (6-, 8- fragments, etc), the Dpnll

132 method has the potential to complement long-range interaction detection.

To further investigate the regulatory significance of the difference in detection range between 4-cutters and HindIII, we overlapped the promoter-interacting genomic regions with

136 regulatory features determined by ATAC-seq for open chromatin and ChIP-seq for histone

137 marks (Supplemental Table 6) in the respective cell types. Promoter-interacting genomic

138 regions detected by 4 -cutter $\mathrm{PCC}$ and $\mathrm{PCHiC}$ were significantly more enriched for active 139 promoter marks (H3K4me3), enhancer marks (H3K27ac and H3K4me1), and regions of open 140 chromatin (ATAC-seq, Figure 2 A and B) compared to HindllI PCHiC, but less enriched for the 
141 H3K36me3 mark that is enriched at the 3' ends of transcriptionally elongating genes and

142 contributes to the composition of heterochromatin ${ }^{15}$. We observed the same enrichment of

143 active chromatin signatures by Dpnll in both blood-derived and tonsil-derived naïve B cells

144 (Figure 2 A), suggesting that this enrichment is not an artifact of donor or tissue of origin, but

145 reflects a real difference between resolutions. Furthermore, to remove the influence of

146 artefactual interactions due to partial digestion in PCC, we filtered out interactions called at less

147 than 4 fragments away from a bait. Importantly, removal of these supra-proximal interactions

148 from the 1 fragment resolution PCC datasets did not impact the enhanced enrichment of

149 regulatory regions in Dpnll PCC libraries compared to Hindll PCHiC libraries, indicating that

150 partial digestion artifacts in PCC libraries do not significantly impair the ability of this technique

151 to capture interactions between gene promoters and regulatory chromatin elements

152 (Supplemental figure 5). Overall, these results suggest that 4-cutter libraries detect functionally

153 relevant regulatory regions than the lower resolution Hindlll-based maps.

One important application of promoter capture data is to discover the effector genes that may be regulated by disease-associated causal variants in relevant tissues, i.e. variant-to-gene

157 mapping. To compare the efficiency of causal variant detection by 4-cutter- and 6-cutter-based 158 approaches, we performed linkage disequilibrium score regression (LDSC) to estimate the 159 heritability content of PIRs in three immune cell types across eight autoimmune and seven 160 psychiatric arbitrarily selected GWAS datasets, and PIRs in cardiomyocytes across three 161 cardiac-related traits (Supplemental Table 6). In general, variants associated with Crohn's 162 disease, ulcerative colitis, and celiac disease were enriched in all three immune cell types, 163 schizophrenia variants were modestly enriched in T and B lymphocytes (Figure 2 C, FDR<0.05,

164 Supplemental Table 7), and variants associated with atrial fibrillation and heart failure were

165 highly enriched in cardiomyocytes (Figure 2 D, FDR<0.05, Supplemental Table 7). Compared

166 to promoter interacting genomic regions derived from HindIII PCHiC, the shorter-range regions 
167 detected by DpnII PCC and Mbol PCHiC were more enriched for heritability of the

168 corresponding traits (Figure 2 C and D). This same trend between distance and heritability

169 could be observed within the HindIII PCHiC datasets themselves, as disease heritability was

170 enriched in the set of Hindlll-based interacting regions located less than 500kb away from

171 promoters, but no significant enrichment was detected for regions beyond 500kb

172 (Supplemental Figure 6 A). Finally, as also observed with feature enrichment, the enhanced

173 heritability enrichment of cell type-relevant traits by high-resolution PIRs was not impacted if

174 potentially artefactual interactions due to partial digestion is filtered from the PCC datasets

175 (Supplemental Figure 6 B). Together, these results demonstrate that high resolution Dpnll and

$176 \mathrm{Mbol}$ maps capture disease-relevant promoter interactions to a greater extent than HindlII-

177 based maps, and importantly, imply that disease-causal variants are most commonly located

178 less than $500 \mathrm{~kb}$ from the promoter of their effector gene.

Overall, we conclude that distinct promoter-chromatin conformation capture techniques and

181 restriction enzymes detect distinct promoter interactomes in the same cell types, owing largely

182 to very different interaction range distributions. HindllI PCHiC maps excel at detecting

183 interactions $>150 \mathrm{~kb}$ to $2 \mathrm{Mb}$ from promoters, but potentially bias investigators away from genes

184 nearest GWAS signals. Conversely, higher resolution PCC and PCHiC maps are more sensitive

185 at detecting highly interactive gene regulatory architectures built from active chromatin regions

186 located relatively proximal $(<150 \mathrm{~kb})$ to gene promoters. High-resolution gene regulatory

187 architectures are also enriched for variants that are strongly associated with relevant common

188 complex traits in given cell types. Moreover, the power of 4-cutter-based capture techniques to

189 detect longer-range interactions can be increased through in silico binning of reads from

190 neighboring fragments, which also eliminates partial digestion artifacts inherent to PCC libraries.

191 Therefore, publicly available promoter capture datasets generated with different restriction 
bioRxiv preprint doi: https://doi.org/10.1101/2020.12.15.422932; this version posted December 15, 2020. The copyright holder for this preprint (which was not certified by peer review) is the author/funder, who has granted bioRxiv a license to display the preprint in perpetuity. It is made available under aCC-BY-NC-ND 4.0 International license.

192 enzymes should not be considered as comparable, even from the same cell types, and

193 investigators should carefully consider which promoter capture approaches or publicly available

194 datasets best fit the goals of their research.

195

196 


\section{Methods}

\section{Promoter capture bait design}

199 Bait design for HindIII PCHiC datasets was downloaded from Open Science Framework 200 (https://osf.io/u8tzp), while Mbol PCHiC bait design was derived from Supplemental Table 9 by

201 Montefiori et $\mathrm{al}^{10}$. Dpnll PCC designs contains both 1-fragment and 4-fragment resolution and 202 were obtained from Array Express (E-MTAB-6862) $)^{3}$. To make bait design comparable across

203 the studies, all baits were re-annotated to genes using bait coordinates overlapping with 204 transcription start site (TSS) from Ensembl v73. The bait design comparison is summarized in 205 Supplemental Table 2.

\section{Promoter capture $\mathrm{C}$ pre-processing and interaction calling}

208 Dpnll PCC libraries from 3 to 4 donors for monocytes, naïve CD4+, naïve B and iPSC cells were pre-processed using the HiCUP pipeline $(\mathrm{v} 0.5 .9)^{16}$, with bowtie2 as aligner and hg19 as

210 the reference genome. Unique captured read pairs from all baited promoters were used for

211 significant promoter interaction calling. Significant promoter interactions at 1-fragment resolution

212 were called using $\mathrm{CHiCAGO}^{13}$ (v1.1.8) with default parameters except for binsize set to 2500.

213 Significant interactions at 4-Dpnll fragment resolution were also called using CHiCAGO with

214 artificial baitmap and rmap files in which Dpnll fragments were concatenated in silico into 4

215 consecutive fragments using default parameters except for removeAdjacent set to False and

216 binsize set to 10000. Interactions with a CHiCAGO score > 5 in either 1 -fragment or 4-fragment

217 resolution were considered as significant interactions. The significant interactions were finally

218 converted to ibed format in which each line represents a physical interaction between

219 fragments.

221 HindIII PCHiC immune datasets were also pre-processed using HICUP by Javierre et al ${ }^{1}$. The 222 summary statistics were extracted from the original paper (Supplemental Table 1). CHiCAGO 
223 R objects were downloaded from Open Science Framework (https://osf.io/u8tzp) and significant

224 interactions were exported using function "exportResults" with CHiCAGO score > 5. The

225 significant interactions for HindIII PCHiC cardiomyocytes were identified by Choy et al ${ }^{17}$ for each

226 replicate independently. The reproducible interactions that are significant in at least two

227 replicates were used for comparison.

229 Mbol PCHiC datasets (cardiomyocytes and iPSC) were pre-processed using HiCUP by 230 Montefiori et $\mathrm{al}^{10}$. The summary statistics were extracted from the original paper (Supplemental

231 Table 1). The significant interactions for HindIII PCHiC were identified for each replicate 232 independently by original paper. The reproducible interactions that are significant in at least two 233 replicates were used for further comparison.

235 The significant interactions from HindIII PCHiC, Dpnll PCC and Mbol PCHiC can be viewed at UCSC browser.

ATAC-seq and histone mark peaks

239 ATAC-seq peaks were called using the ENCODE ATAC-seq pipeline

240 (https://www.encodeproject.org/atac-seq/). Briefly, pair-end reads from all replicates for each

241 cell type were aligned to hg19 genome using bowtie2, and duplicate reads were removed from

242 the alignment. Aligned tags were generated by modifying the reads alignment by offsetting $+4 \mathrm{bp}$

243 for all the reads aligned to the forward strand, and $-5 \mathrm{bp}$ for all the reads aligned to the reverse

244 strand. Narrow peaks were called independently for pooled replicates for each cell type using

245 macs2 (-p 0.01 --nomodel --shift -75 --extsize 150 -B --SPMR --keep-dup all --call-summits) and

246 ENCODE blacklist regions (wgEncodeDacMapabilityConsensusExcludable.bed.gz) were

247 removed from called peaks. The reference peaks for each cell type were merged peaks present 248 in at least half of replicates. 
250 Histone mark peaks were downloaded as processed bed files from The BLUEPRINT Data

251 Analysis Portal ${ }^{18}$ (Supplemental Table 6). The reference peaks for each cell type and tissue

252 were obtained by merging replicates and selecting peaks present in at least half of replicates.

253 The hg38 coordinates were finally converted to hg19 coordinates using UCSC liftOver.

255 Feature enrichment

256 When Chicago objects were available (Naïve CD4+, Naïve B and Monocytes), the feature 257 enrichment was performed independently for each cell type and resolution, using 258 "peakEnrichment4Features" function from CHiCAGO (v1.1.8) and plotted with ggplot2. For the 259 samples without Chicago objects (cardiomyocetes), PIRs identified by significant interactions 260 was compared to PIRs identified by non-significant interactions by overlapping with chromatin 261 features in corresponding cell type using Locus Overlap Analysis (LOLA) ${ }^{20}$. The odds ratio is 262 determined by Fisher's exact test for both cutters independently.

264 Partitioned heritability LD score regression enrichment analysis

265 Partitioned heritability LD Score Regression ${ }^{19}$ (v1.0.0) was used to identify heritability 266 enrichment with GWAS summary statistics and PIRs characterized by Chicago. The baseline 267 analysis was using performed LDSCORE data

268 (https://data.broadinstitute.org/alkesgroup/LDSCORE) with LD scores, regression weights, and 269 allele frequencies from 1000G Phase1. The summary statistics were downloaded using the links 270 and reference provided in Supplemental Table 6. The Partitioned LD score regression 271 annotations were generated using the coordinates of non-bait PIRs independently for each cell 272 type and cutters. Finally, the cell-type-specific partitioned LD scores were compared to baseline 273 LD scores to measure enrichment fold change and enrichment p-value were adjusted using 
bioRxiv preprint doi: https://doi.org/10.1101/2020.12.15.422932; this version posted December 15,2020 . The copyright holder for this preprint (which was not certified by peer review) is the author/funder, who has granted bioRxiv a license to display the preprint in perpetuity. It is made available under aCC-BY-NC-ND 4.0 International license.

274 FDR across all comparisons. The details of the LDSC enrichment results are provided in

275 Supplemental Table 7. 


\section{References}

277 1. Javierre, B. M. et al. Lineage-Specific Genome Architecture Links Enhancers and Non-

278 coding Disease Variants to Target Gene Promoters. Cell 167, 1369-1384.e19 (2016).

279 2. Thiecke, M. J. et al. Cohesin-Dependent and -Independent Mechanisms Mediate

280 Chromosomal Contacts between Promoters and Enhancers. Cell Rep. 32, 107929 (2020).

281 3. Chesi, A. et al. Genome-scale Capture C promoter interactions implicate effector genes

282 at GWAS loci for bone mineral density. Nat. Commun. 10, 1260 (2019).

283 4. Su, C. et al. Mapping effector genes at lupus GWAS loci using promoter Capture-C in

284 follicular helper T cells. Nat. Commun. 11, 1-17 (2020).

285 5. Song, M. et al. Mapping cis-regulatory chromatin contacts in neural cells links

286 neuropsychiatric disorder risk variants to target genes. Nat. Genet. 51, 1252-1262 (2019).

287 6. Hughes, J. R. et al. Analysis of hundreds of cis-regulatory landscapes at high resolution

288 in a single, high-throughput experiment. Nat. Genet. 46, 205-212 (2014).

289 7. Schoenfelder, S., Javierre, B. M., Furlan-Magaril, M., Wingett, S. W. \& Fraser, P.

Promoter capture Hi-C: High-resolution, genome-wide profiling of promoter interactions. J.

$291 \quad$ Vis. Exp. (2018). doi:10.3791/57320

292 8. Freire-Pritchett, P. et al. Global reorganisation of cis-regulatory units upon lineage 293 commitment of human embryonic stem cells. Elife 6, (2017).

294 9. Rubin, A. J. et al. Lineage-specific dynamic and pre-established enhancer-promoter 295 contacts cooperate in terminal differentiation. Nat. Genet. (2017). doi:10.1038/ng.3935

296 10. Montefiori, L. E. et al. A promoter interaction map for cardiovascular disease genetics.

$297 \quad$ Elife 7, 1-35 (2018).

298 11. Shi, L. et al. IL-1 Transcriptional Responses to Lipopolysaccharides Are Regulated by a 299 Complex of RNA Binding Proteins. J. Immunol. 204, 1334-1344 (2020).

300 12. Natarajan, A., Yardimci, G. G., Sheffield, N. C., Crawford, G. E. \& Ohler, U. Predicting 301 cell-type-specific gene expression from regions of open chromatin. Genome Res. (2012). 
303 13. Cairns, J. et al. CHiCAGO: Robust detection of DNA looping interactions in Capture Hi-C data. Genome Biol. 17, 1-17 (2016).

14. Rocha, P. P., Raviram, R., Bonneau, R. \& Skok, J. A. Breaking TADs: Insights into hierarchical genome organization. Epigenomics (2015). doi:10.2217/epi.15.25

15. Chantalat, S. et al. Histone $\mathrm{H} 3$ trimethylation at lysine 36 is associated with constitutive and facultative heterochromatin. Genome Res. (2011). doi:10.1101/gr.118091.110

16. Wingett, S. et al. HiCUP: Pipeline for mapping and processing Hi-C data. F1000Research (2015). doi:10.12688/f1000research.7334.1

311 17. Choy, M. K. et al. Promoter interactome of human embryonic stem cell-derived cardiomyocytes connects GWAS regions to cardiac gene networks. Nat. Commun. (2018). doi:10.1038/s41467-018-04931-0

314 18. Fernández, J. M. et al. The BLUEPRINT Data Analysis Portal. Cell Syst. (2016). doi:10.1016/j.cels.2016.10.021

19. Finucane, H. K. et al. Partitioning heritability by functional annotation using genome-wide association summary statistics. Nat. Genet. 47, 1228-1235 (2015). regulatory elements in R and Bioconductor. Bioinformatics 32, 587-589 (2015). 


\section{FIGURE LEGENDS}

322 Figure 1. Interaction range detection difference results in low overlap between Dpnll and HindIII.

323 A. Distance ranges of intra-chromosomal interaction In Dpnll PCC vs. Hindlll PCHiC (panels 1-

324 3) and Mbol PCHiC vs. Hindlll PCHiC (panel 4). The distance between mid-points of bait

325 fragment and PIR was plotted in boxplot for significant cis-interactions (CHiCAGO score > 5).

326 The upper whisker, upper hinge, middle line, middle dot, down hinge and down whisker indicate

$32795 \%, 75 \%$, median, mean, 25\% and 5\% percentile. B. The number of significant cis-interaction

328 across different distance between bait and PIR. The significant cis-interactions in Dpnll PCC vs.

329 HindIII PCHiC were grouped based the binned distance (250k intervals) between mid-points of

330 bait fragment and PIR. Interaction number within each group was plotted. The inset depicts

331 comparative distance interaction distributions of Mbol PCHiC vs. Hindlll PCHiC. C. Venn

332 diagram of genes annotated in DpnII PCC, Mbol PCHiC, and HindIII PCHiC datasets. Genes

333 were annotated when the bait of a significant interaction harbors its TSS. Regardless of PIR

334 overlaps, shared (light green), Dpnll-specific (dark green), Mbol-specific (red), and HindIII-

335 specific (blue) genes were determined based on whether corresponding baits have at least one

336 significant distal interaction in both Dpnll and HindIII, Mbol and HindIII, or DpnII and Mbol

337 datasets. D. Distance difference between interactions involving cutter-specific and shared genes.

338 The interaction distance distribution measured by each method (x-axis) was calculated for

339 cutter-specific gene sets (blue) or shared gene sets (red). The labeled p-value was determined

340 by two-tailed wilcoxon rank sum test. E. Example of SERBP1 interacting region range difference

341 between DpnII PCC and HindIII PCHiC in naïve B cells. The Dpnll and HindIII baits for SERBP1

342 were overlapped. Only significant interactions (CHiCAGO score > 5) were shown with arcs in

343 either Dpnll PCC (red) and HindIII PCHiC (blue).

345 Figure 2. Enrichment of regulatory DNA and heritability of immune and psychiatric traits by

346 PIRs from immune cells. A. Enrichment of regulatory regions by promoter-interacting regions. 
347 The number of PIR overlapped with open chromatin and histone binding regions were

348 calculated in both significant interactions and randomly distance-matched non-significant

349 interactions. The enrichment fold change is measure by the ratio of PIR number between

350 significant and non-significant interactions. Mean $\square \pm \square 95 \% \mathrm{Cl}$ is depicted across 100 draws of

351 non-significant interactions. B. Enrichment of regulatory regions by promoter-interacting regions

352 in cardiomyocytes. The odds ratio is determined by Locus Overlap Analysis (LOLA) ${ }^{20}$ for both

353 cutters independently. It compares PIRs identified by significant interactions to PIRs identified

354 by non-significant interactions using Fisher's exact test. C. Heritability enrichment of

355 autoimmune and psychiatric disorders by PIRs in immune cells. Partitioned LD score regression

356 was performed independently in three immune cell types (Monocyte, naïve B and CD4 T cells)

357 across 15 GWAS studies and PIRs detected by different cutters. Enrichment of heritability (blue)

358 was measured in PIRs compared to baseline, and p-value was adjusted with FDR (orange). D.

359 Heritability enrichment of GWAS traits by PIRs in cardiomyocytes. Partitioned LD score

360 regression was performed independently across 18 GWAS studies and PIRs detected by

361 different cutters. Enrichment of heritability was measured in PIRs compared to baseline and

362 labeled with heatmap. The significant enrichment was indicated by stars $\left({ }^{*} P<0.05\right.$, $\left.{ }^{*} P<0.01\right)$.

363 Details can be found in Supplemental Table 7.

365 Supplemental Figure 1. Difference between PCC and PCHiC. The percentage of cis-capture 366 read pair count $(\mathbf{A})$ and cis significant interaction number $(\mathbf{B})$ across different fragment intervals 367 between bait and PIR. PCC (red) and PCHiC (green) libraries were both prepared by 4-cutters 368 for iPSC cells. In silico 4-fragment resolution is indicated by triangle.

370 Supplemental Figure 2 Resolution comparison between Dpnll/Mbol and Hindlll design. A.

371 Fragment length of Dpnll/Mbol and Hindlll by in silico digesting hg19 genome. Dpnll and Mbol 372 recognized the same sequences (^GATC) while HindIII recognize 6-bp sequence ( ${ }^{\wedge}$ AGCTT). 
373 Fragment length was plotted in boxplot for all fragments (left) and bait fragments (right). The

374 upper whisker, upper hinge, middle line, middle dot, down hinge and down whisker indicate 95\%

375 percentile, $75 \%$ percentile, median, mean, 25\% percentile and 5\% percentile. B. Example of

376 bait annotation precision difference between DpnII PCC and HindIII PCHiC at ENTHD1 and

377 GRAP2. Black bars indicate the baits. Two baits in Dpnll (red arcs) design represents ENTHD1

378 and GRAP2 independently while these two genes are baited by the same fragment in HindIII

379 (blue arcs) design.

381 Supplemental Figure 3. The normalized read count of significant cis-interaction across

382 distance between bait and PIR. The significant cis-interactions were grouped based the binned

383 distance (250k intervals) between mid-points of bait fragment and PIR. The reads were

384 normalized by total "reference" read count for each cell. The median normalized read count

385 within each group were plotted.

387 Supplemental Figure 4. Bi-directional (reproducible) bait-to-bait interactions detected by Dpnll

388 PCC and HindIII promoter capture HiC. A. Bait-to-bait interaction self-reproducibility. The self-

389 reproducibility was measured by ratio between bi-directional and all bait-to-bait significant

390 interactions (CHICAGO $\geq 5)$. B. Distribution of bait-specific and PIR-specific scaling factors. The

391 scaling factors were extracted from CHICAGO R objects across all interactions. The average of

392 scaling factors per fragment among all cell types are plotted for distribution.

394 Supplemental Figure 5. Regulatory feature enrichment of PIRs by filtered out interactions

395 called at less than 4 fragments away from a bait. The number of PIR overlapped with open

396 chromatin and histone binding regions were calculated in both significant interactions and

397 randomly distance-matched non-significant interactions. The enrichment fold change is measure 
398 by the ratio of PIR number between significant and non-significant interactions. Mean $\square \pm \square 95 \%$

$399 \mathrm{Cl}$ is depicted across 100 draws of non-significant interactions.

400

401 Supplemental Figure 6. LDSC enrichment in CEL, CRO and UC in immune cells. A.

402 Heritability of autoimmune traits is enriched at PIRs within 500kbp from gene promoter.

403 Partitioned LD score regression was performed independently for PIRs within and beyond

$404500 \mathrm{kbp}$ from HindIII PCHiC data sets. Enrichment of heritability (blue bar) was measured in

405 PIRs of given distance compared to baseline, and p-value was adjusted with FDR (orange

406 point). B. Higher heritability enrichment of PIRs in Dpnll PCC is not impacted by artefactual

407 interactions from the partial digestions. Partitioned LD score regression was performed

408 independently for a set of Dpnll PCC PIRs from which interaction with less than 2 to 20

409 fragments away were removed. The heritability enrichment of HindIII PCHiC PIRs is indicated

410 by black dashed lines. 
415 Supplemental Table 1: Hicup pre-processing of HindIII PCHiC, Dpn II PCC and Mbol

416 PCHiC libraries used in this study.

418 Supplemental Table 2: Bait design and annotation of HindIII PCHiC, Dpn II PCC and Mbol 419 PCHiC

421 Supplemental Table 3: Comparison of total and significant interaction calls from 422 CHiCAGO.

424 Supplemental Table 4: The pairwise comparison of significant cis interactions among 425 HindIII PCHiC, Dpn II PCC and Mbol PCHiC libraries within the same cell type.

427 Supplemental Table 5: The pairwise comparison of identified gene-PIR interactions 428 among HindIII PCHiC, Dpn II PCC and Mbol PCHiC libraries within the same cell type.

430 Supplemental Table 6: External resource for chromatin feature and GWAS summary 431 statistic. 
bioRxiv preprint doi: https://doi.org/10.1101/2020.12.15.422932; this version posted December 15, 2020. The copyright holder for this preprint Figu (which was not certified by peer review) is the author/funder, who has granted bioRxiv a license to display the preprint in perpetuity. It is made

A.
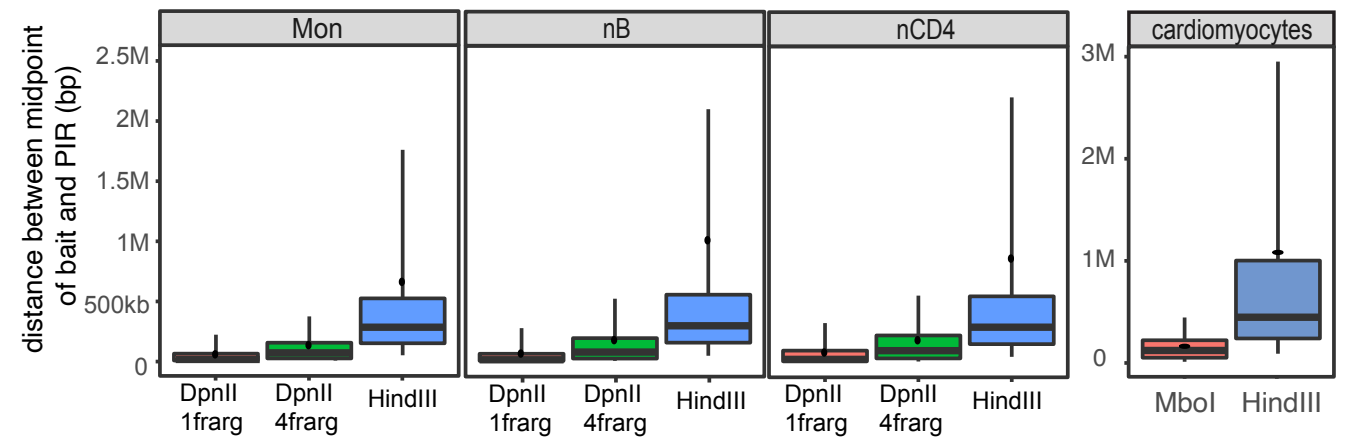

B.
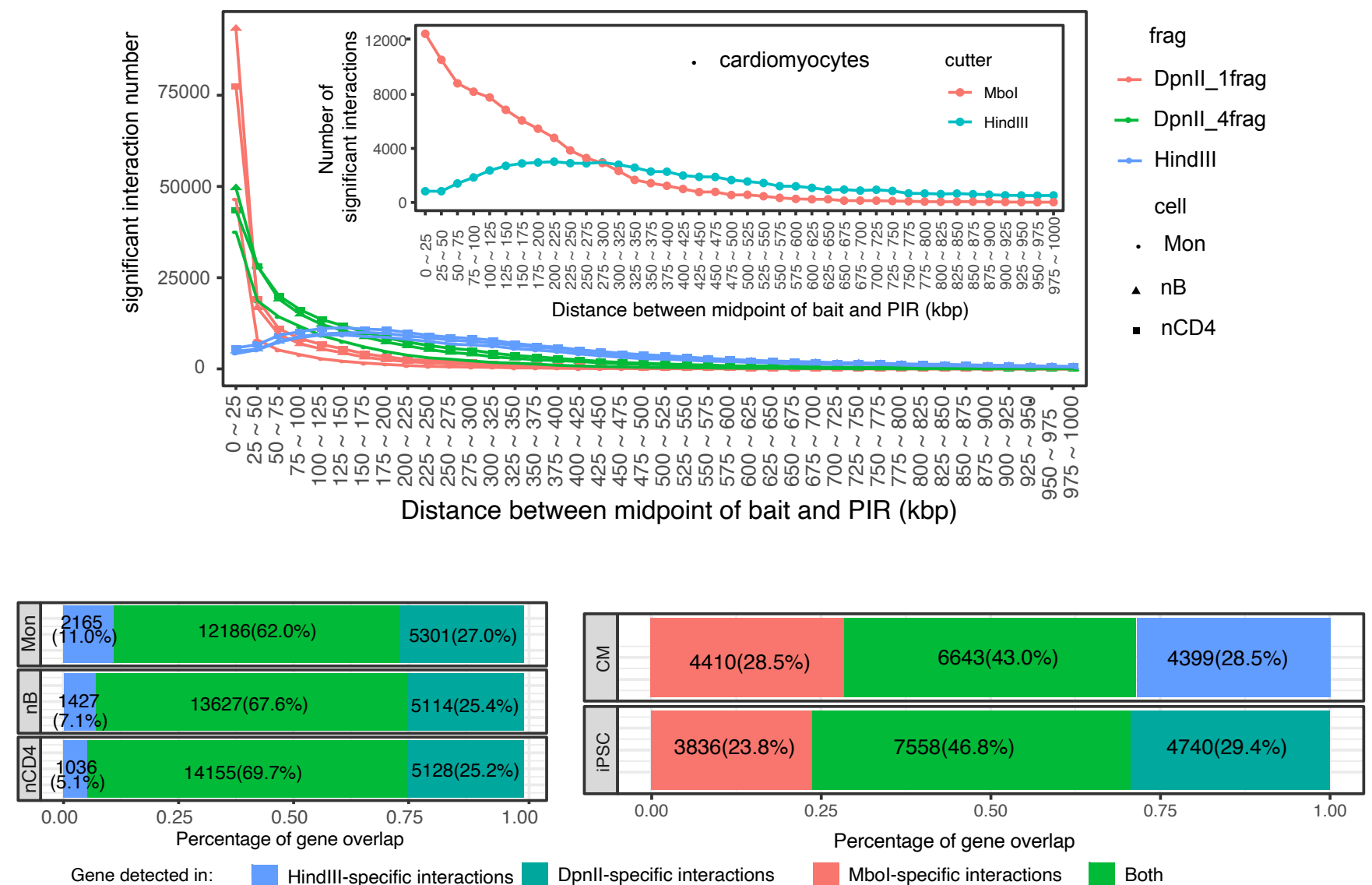

c.

Dpnll-specific interactions

Mbol-specific interactions

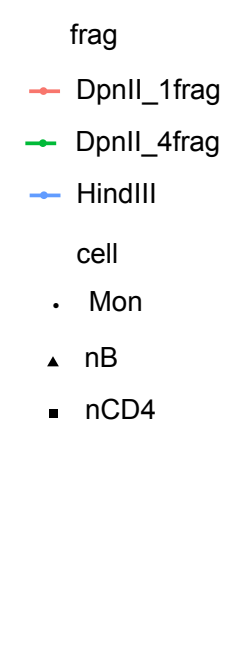

D.

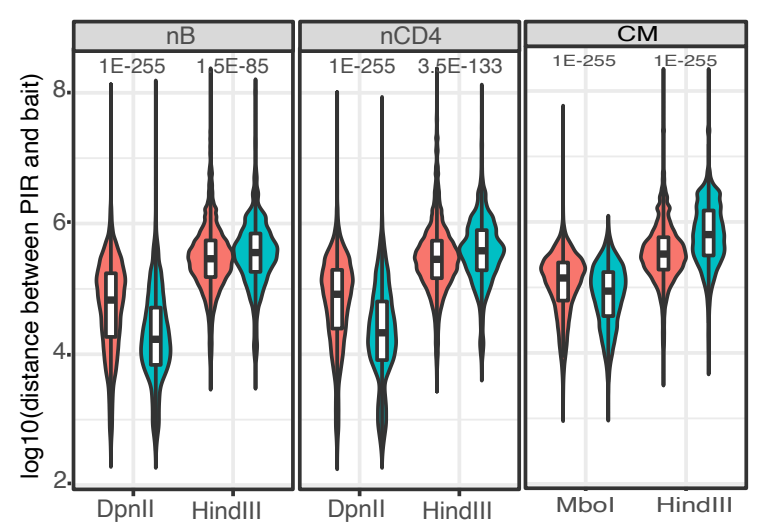

Gene detected in: 官 Both 芦 cutter-specific interactions
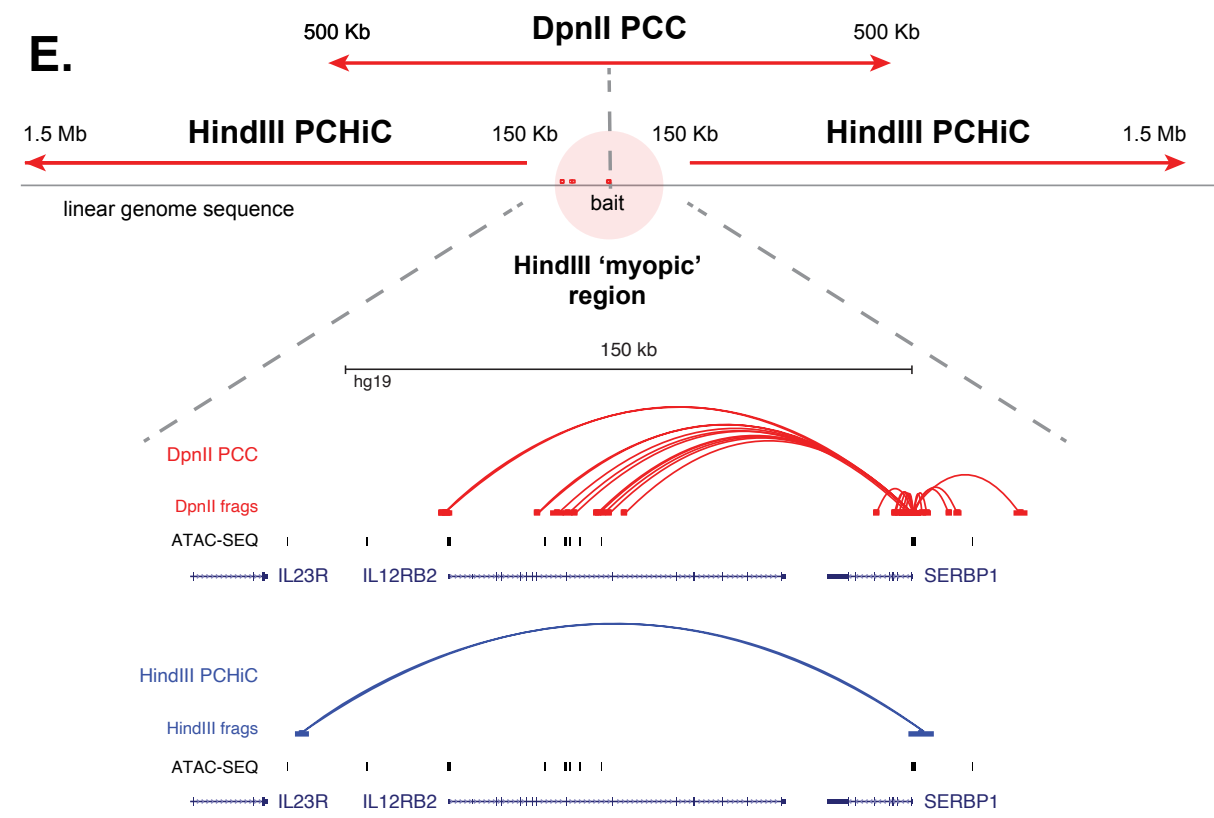
Figure 2

A.

bioRxiv preprint doi: https://doi.org/10.110 12020.12 .15 .422932 ; this version posted December 15, 2020. The copyright holder for this preprint (which was not certified by peer review) is the author/funder, who has granted bioRxiv a license to display the preprint in perpetuity. It is made nB_venous_blood. available under aCC-BY-NC-ND 4.0 International license.

Mon_venous_blood

nB_venous_blood

nB_tonsil

Mon_venous_blood

nCD4_blood

nB_venous_blood

$n B$ tonsil

Mon_venous_blood
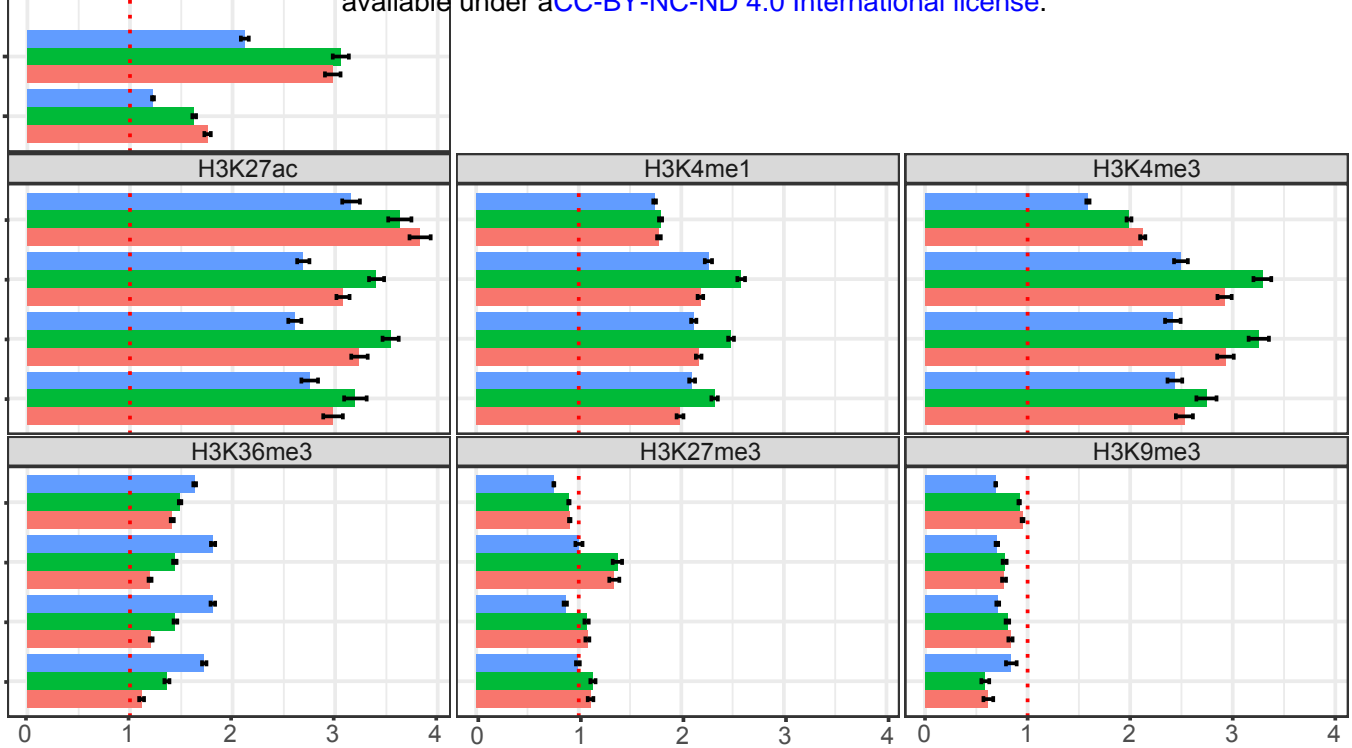

frag

Dpn1frag

Dpn4frag

HindIII

B.

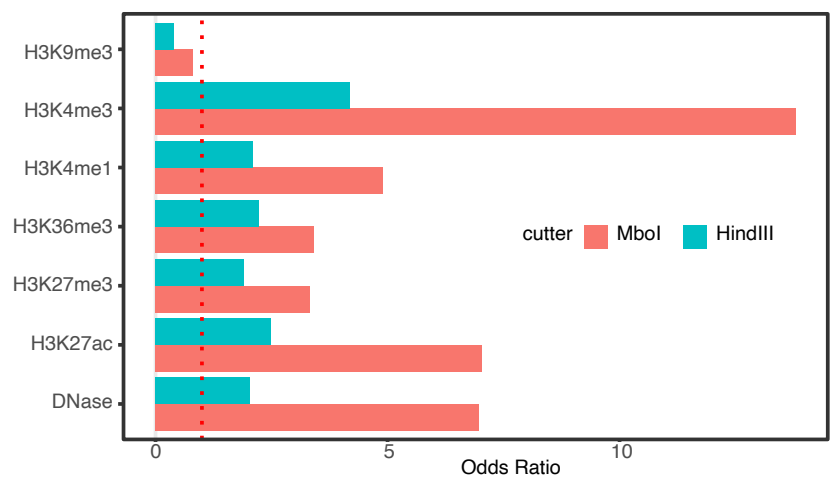

C.
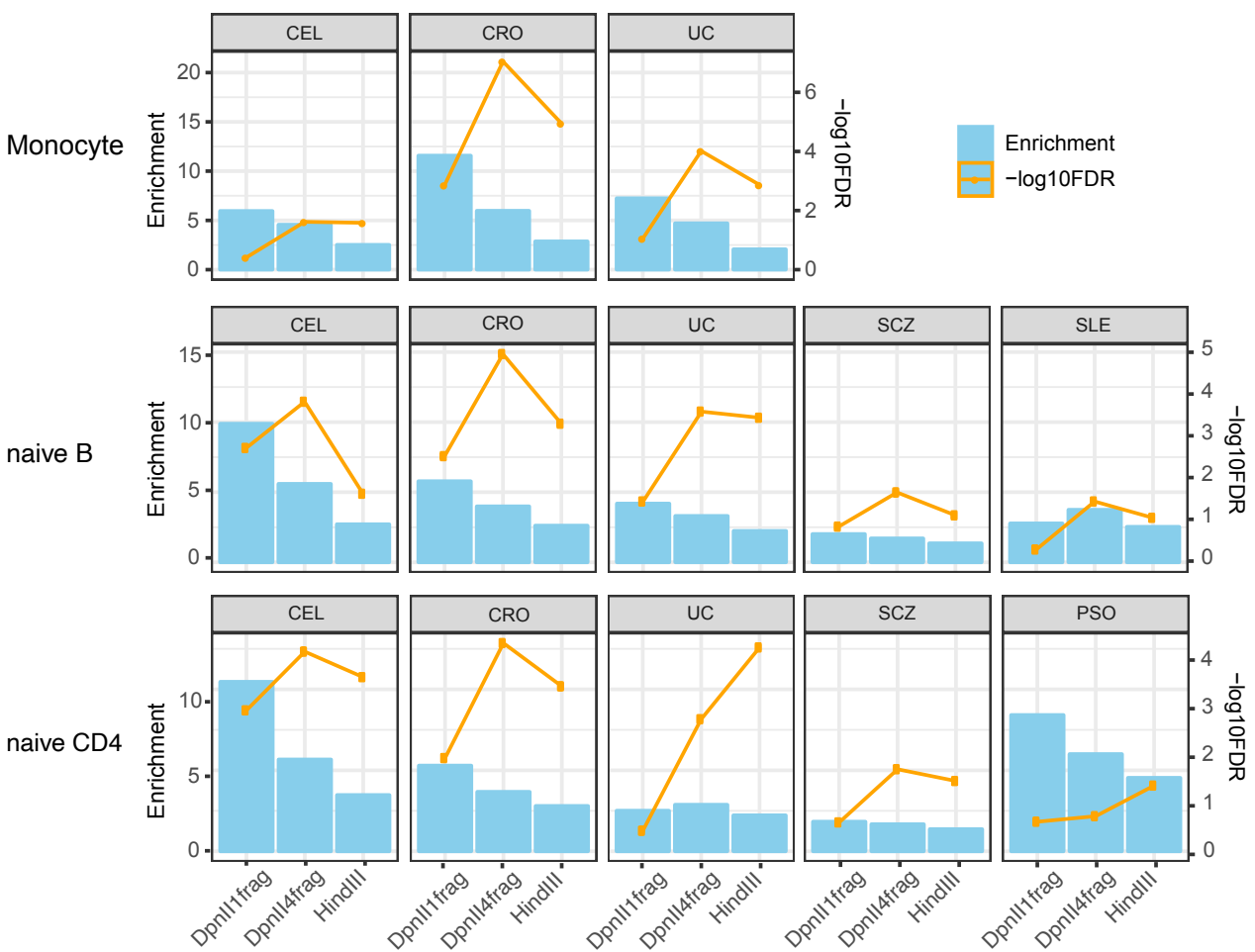

D.

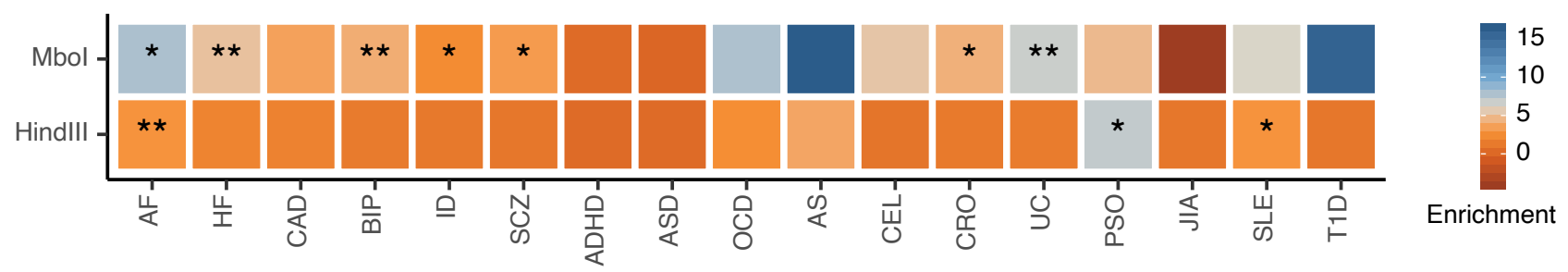

